\title{
Lower than average HDL cholesterol efflux capacity in Lithuanian population
}

Sandra Kutkiene ${ }^{1,2,3^{*}}$, Zaneta Petrulioniene ${ }^{1,2,3}$, Dovile Karciauskaite ${ }^{1,3,4}$, Aleksandras Laucevicius ${ }^{1,2,3}$, Gabija Matuzevicienè $\dot{1}^{1,3}$, Justina Staigyte ${ }^{1,3}$, Akvile Saulyte Mikulskiene ${ }^{1,3}$, Urte Gargalskaite ${ }^{3}$, Egle Skiauteryte ${ }^{1,3}$ and Milda Kovaite ${ }^{3}$

\begin{abstract}
Background: The aim of our study was to evaluate high-density lipoprotein cholesterol (HDL-C) efflux capacity in healthy controls and patients with severe dyslipidemia. Evaluation of HDL function may be beneficial for better understanding of cardiovascular diseases, as well as for taking actions to minimize residual cardiovascular risk.

Methods: During 2016-2017 a total of 93 participants - 48 (51.6\%) women and 45 (48.4\%) men - were included in this cross-sectional study. Data of 45 (48.4\%) participants with severe dyslipidemia (SD) and 48 (51.6\%) controls without dyslipidemia was used for statistical analysis. Total lipid panel, concentration of lipoprotein (a) and apolipoproteins were measured, data about cardiovascular risk factors were collected and detailed evaluation of $\mathrm{HDL}-\mathrm{C}$ quality was performed for all patients.

Results: Increased HDL-C concentration was associated with higher ApoA1 ( $r=0.866$ in controls, $r=0.63$ in SD group), ApoA2 ( $r=0.41$ in controls, $r=0.418$ in SD group) and LDL-C concentrations ( $r=-0.412$ in SD group), lower ApoE ( $r=-0.314$ in SD group) and TG concentrations ( $r=-0.38$ in controls, $r=-0.608$ in SD group), lower ApoB/ ApoA1 ratio ( $r=-0.567$ in control group), below average HDL-C efflux capacity ( $r=-0.335$ in SD group), lower BMl ( $r=-0.327$ in controls, $r=-0.531$ in SD group) and abdominal circumference ( $r=-0.309$ in women with SD). Below-average HDL-C efflux capacity was found in $67.7 \%(N=63)$ of participants. It was more often found among patients with normal weight or BMI $30-31 \mathrm{~kg} / \mathrm{m} 2$. HDL-C efflux capacity was inversely associated with HDL-C concentration $(r=-0.228)$.
\end{abstract}

Conclusion: Abnormal HDL function may be associated with residual cardiovascular risk in Lithuanian population.

Keywords: HDL cholesterol efflux capacity, Severe dyslipidemia, HDL function, Residual risk

\section{Background}

Cardiovascular disease accounts for $65 \%$ of deaths among women and $48 \%$ among men [1]; according to EUROSTAT, Lithuania recorded the highest rate of death due to ischemic heart disease in Europe [2]. According to the Lithuanian High Cardiovascular Risk (LitHiR) primary prevention program, the prevalence of any type of dyslipidemia in Lithuania is high $-89.7 \%$ [3], although only $13.7 \%$ of middle-aged Lithuanians have low HDL-C [4]. HDL is responsible for many actions related to maintaining arterial

\footnotetext{
* Correspondence: Sandra.kutkiene@santa.It

${ }^{1}$ Faculty of Medicine, Vilnius University, Santariškių 2, LT-08661 Vilnius, Lithuania

${ }^{2}$ Faculty of Medicine, Clinic of Cardiac and Vascular Diseases, Vilnius University, Vilnius, Lithuania

Full list of author information is available at the end of the article
}

wall homeostasis, such as reverse cholesterol transport, anti-inflammatory, antioxidative, anti-apoptotic, antiinfectious, anti-thrombotic and vasodilatory effects $[5,6]$. HDL-C cardio-protective role has been supported by large population studies $[7,8]$. It was estimated that by increasing HDL-C concentration by $0.026 \mathrm{mmol} / \mathrm{l}(1 \mathrm{mg} / \mathrm{dL})$, cardiovascular disease risk decreases by $2 \%$ in men and $3 \%$ in women [9]. On the other hand, some novel studies show attenuated relationship between low HDL-C and coronary heart disease (CHD) risk [10]. The relationship between HDL-C levels and CHD incidence does not seem to be causal, both according to Mendelian randomization studies [11], and based on the fact that drugs targeted to increase HDL-C concentration failed to show benefit in randomized controlled trials [12, 13], [14]. In addition, Danish

(c) The Author(s). 2019 Open Access This article is distributed under the terms of the Creative Commons Attribution 4.0 International License (http://creativecommons.org/licenses/by/4.0/), which permits unrestricted use, distribution, and 
population studies revealed that both low and high HDL-C concentration were associated with higher all cause and cardiovascular mortality [15]. Individuals with certain genetic variants and very high HDL-C concentration may as well develop atherosclerosis, which could be explained by abnormal HDL function [16]. Better understanding of HDL function would be of great benefit, as impaired HDL function may be associated with residual cardiovascular risk. There are multiple laboratory assays for evaluation of various cardio-protective effects of HDL. HDL-C efflux capacity assay evaluates how HDL removes cholesterol from cells, including macrophages, through ABCA1, and represents a major athero-protective role of HDL particles, as shown in vivo [17] and in real life data, since HDL-C efflux capacity is significantly and inversely associated with incident coronary heart disease events, independent of several established cardiovascular risk factors [18]. The aim of our study was to evaluate HDL-C efflux capacity in healthy participants and patients with severe dyslipidemia.

\section{Methods}

During 2016-2017 a total of 93 participants (48 (51.6\%) women and $45(48.4 \%)$ men) from 18 up to 60 years of age with severe dyslipidemia (SD) or no lipid abnormality (control group) without possible causes of secondary dyslipidemia and evident cardiovascular disease were included in this cross-sectional study. The study was approved by the Local Research Ethics Committee, and written informed consent was obtained from each participant before including them into the study. Data of 45 (48.4\%) participants with severe dyslipidemia (SD) and 48 (51.6\%) controls without dyslipidemia, overt cardiovascular disease and disorders that may cause secondary dyslipidemia, were collected and detailed evaluation of the HDL-C quality was performed.

$\mathrm{SD}$ was defined as serum total cholesterol $\geq 7.5 \mathrm{mmol} / \mathrm{L}$ (290 mg/dL), or low-density-lipoprotein cholesterol (LDLC) $\geq 6 \mathrm{mmol} / \mathrm{L}(232 \mathrm{mg} / \mathrm{dL})$. Patients with overt cardiovascular disease and possible secondary dyslipidemia were not included into the study (uncontrolled hypothyroidism, diabetes mellitus, nephrotic syndrome, renal insufficiency, cholestasis, viral hepatitis, liver cirrhosis, alcoholism, anorexia, pregnancy, terminal stage cancer and any terminal). Controlled thyroid dysfunction and diabetes mellitus diagnosed later than dyslipidemia were not considered as exclusion criteria. Only subjects without dyslipidemia, evident cardiovascular disease (myocardial infarction, unstable angina, stable angina with positive cardiac stress test, coronary artery pathology identified during cardiac catheterization or coronary computed tomography angiography, coronary artery bypass surgery, percutaneous coronary intervention), cerebrovascular disorder (previous acute ischemic or hemorrhagic stroke, diagnosed stenosis of carotid arteries), peripheral artery disease (acute ischemic syndromes, chronic limb ischemia, aortic aneurysm), disorders that may impact concentrations of blood lipids were included in control group of the study. If patient had SD and treatment with statins or other drugs was provided but lipid profile was still abnormal (fulfilling criteria of severe hypercholesterolemia), patients were included into the study. We did not differentiate patients into SD without treatment group or SH with insufficient treatment.

Total cholesterol, LDL-C, HDL-C, triglycerides (TG), apolipoprotein A1 (ApoA1), apolipoprotein A2 (ApoA2), apolipoprotein $\mathrm{B}(\mathrm{ApoB})$, apolipoprotein $\mathrm{E}(\mathrm{ApoE})$ and lipoprotein (a) ( $\mathrm{Lp}(\mathrm{a})$ ) concentrations, as well as ApoB/ ApoA1 ratio were measured. All the tests were carried out in the morning and participants were advised not to eat for at least $12 \mathrm{~h}$. Data about cardiovascular risk factors, such as arterial hypertension, smoking, alcohol consumption, familial history of CHD and diabetes mellitus in first degree relatives, insufficient physical activity and body mass index (BMI) were collected.

While assessing the BMI, the following groups were identified: ideal -22 , normal $-20-25$ for men and 18.5-24 for women, overweight $-25-29.9$, obese $-30-40$ and severely obese $->40$.

Arterial hypertension was considered as systolic blood pressure $\geq 140 \mathrm{mmHg}$ and/or diastolic blood pressure $\geq$ $90 \mathrm{mmHg}$, or the diagnosis of hypertension was documented in a medical record.

Insufficient physical activity was described as exercises less than 45 min 3 times a week.

HDL-C efflux capacity was evaluated by using Cholesterol efflux fluorometric assay kit (BioVision, Inc., CA, USA) according to manufacturer's protocol. As HDL cholesterol efflux capacity assay does not have standardized reference range, HDL cholesterol efflux was calculated and categorized into tertiles - below average, average and above average.

\section{Statististics}

Categorical variables were described through frequencies (\%) and continuous variables were expressed by means and standard deviations (SD). Continuous variables were compared using the Kruskal-Wallis univariate analysis of variance (ANOVA). Categorical variables were compared using the Chi-square test or Fisher exact test. In order to assess the linear association between characteristics, correlation analysis was performed and Spearman's correlation coefficient $(r)$ was applied. Correlation was defined as weak $(\mathrm{r}<0.3)$, moderate $(0.3 \leq \mathrm{r} \leq 0.7)$ or strong $(\mathrm{r}>$ $0.7)$. A $p$-value of $<0.05$ was considered significant. Statistical analyses were performed using SPSS 23.0 (SPSS Inc., Chicago, Illinois, US). 


\section{Results}

\section{General characteristics}

The baseline characteristics of the participants are provided in Table 1 . There was a significant age difference in the whole study population between participants in SD and control groups $(50.53 \pm 1.11$ years vs. $47.04 \pm 0.76$ years, $p=0.001)$. More patients with severe dyslipidemia had arterial hypertension ( $44.4 \%$ vs. $20.8 \%, p=0.015)$, and family history of CHD (64.4\% vs. $12.5 \%, p<0.001)$, compared to control group (Table 1).

\section{HDL-C concentration}

No significant difference in HDL-C concentration was found between control group and SD group (1.26 \pm 0.32 $\mathrm{mmol} / \mathrm{l}$ vs. $1.30 \pm 0.04 \mathrm{mmol} / \mathrm{l}, \quad p=0.975)$. Decreased HDL-C $(<1.2 \mathrm{mmol} / \mathrm{l}(46 \mathrm{mg} / \mathrm{dL})$ in women and $<1$ $\mathrm{mmol} / \mathrm{l}(39 \mathrm{mg} / \mathrm{dL})$ in men [19]) was found more frequently in participants with SD than in healthy controls (31\% $(N=14)$ vs. $14.6 \%(N=7), p=0.004)$. We found $70.2 \%(n=33)$ of decreased HDL-C concentrations in the SD group. Gender-specific analysis showed that more men with SD had decreased HDL-C $(36.4 \%, n=8)$ compared to the control group (13.6\%, $n=3), p=0.013$, while no significant difference in HDL-C levels between women with SD $(26 \%, n=6)$ and healthy women $(12.5 \%$, $n=3)$ was found $(p=0.118)$.
A weak but significant correlation between age and HDL-C concentration was found $(r=0.180, p=0.008)$. It was estimated that chances of severe dyslipidemia are approximately two times higher (OR 2.433, CI:1.366-4.334) when $\mathrm{HDL}-\mathrm{C}<1.19 \mathrm{mmol} / \mathrm{l} \quad(46 \mathrm{mg} / \mathrm{dL})$ (sensitivity $46.4 \%$, specificity $-73.8 \%$, area under the curve $-2.6 \%$ ).

For further analysis and in order to define increased, normal and decreased HDL-C concentration we calculated 33th percentiles, which divided all measurements into three equal parts. Decreased HDL-C concentration was defined as $<1.08 \mathrm{mmol} / \mathrm{l}(42 \mathrm{mg} / \mathrm{dL})$, normal HDL-C concentration $1.08-1.4 \mathrm{mmo} / \mathrm{l}(42-54 \mathrm{mg} / \mathrm{dL})$ and increased $\mathrm{HDL}-\mathrm{C}$ concentration is $>1.40 \mathrm{mmol} / \mathrm{l}(>54 \mathrm{mg} / \mathrm{dL})$.

The majority of the participants $(67.7 \%, N=64)$ had normal value of HDL-C, irrespective of their SD status. Decreased HDL-C concentration was found in $14.0 \%$ $(N=13)$ of the participants. Increased HDL-C concentration was found in $18.3 \%(N=17)$ of the subjects, more often in women than men $(27 \% N=13$ vs. $8.9 \% N=4$, $p=0.015$ ) (Fig. 1). There was no significant difference in HDL-C concentration in different age groups.

\section{Correlation between HDL-C concentration and other cardiovascular risk factors}

Normal HDL-C concentration was more often found in participants with class I obesity $(p=0.02)$ (Fig. 2) and those who consumed alcohol, in comparison with those

Table 1 The baseline characteristics and trends of cardiovascular risk factors of the study population $(n=93)$

\begin{tabular}{|c|c|c|c|c|c|c|c|}
\hline \multirow[t]{3}{*}{ Characteristics } & \multirow{2}{*}{\multicolumn{2}{|c|}{$\begin{array}{l}\text { All patients } \\
n=93\end{array}$}} & \multirow{2}{*}{\multicolumn{2}{|c|}{$\begin{array}{l}\text { Severe dyslipidemia group } \\
n=45\end{array}$}} & \multirow{2}{*}{\multicolumn{2}{|c|}{$\begin{array}{l}\text { Control group } \\
n=48\end{array}$}} & \multirow[t]{3}{*}{$p$} \\
\hline & & & & & & & \\
\hline & Mean & SD & Mean & SD & Mean & SD & \\
\hline Age (years) & 48.73 & 6.60 & 50.53 & 1.11 & 47.04 & 0.76 & 0.001 \\
\hline $\mathrm{TC}(\mathrm{mmol} / \mathrm{l})$ & 6.16 & 2.32 & 7.93 & 0.32 & 4.51 & 0.08 & $<0.001$ \\
\hline LDL-C (mmol/l) & 4.05 & 2.06 & 5.42 & 0.33 & 2.77 & 0.07 & $<0.001$ \\
\hline HDL-C (mmol/l) & 1.28 & 0.32 & 1.26 & 0.05 & 1.30 & 0.04 & 0.975 \\
\hline TG (mmol/l) & 1.77 & 1.82 & 2.63 & 0.34 & 0.95 & 0.06 & $<0.001$ \\
\hline ApoA1 (mmol/l) & 1.66 & 0.25 & 1.68 & 0.04 & 1.64 & 0.03 & 0.321 \\
\hline ApoB (mmol/l) & 1.10 & 0.49 & 1.47 & 0.07 & 0.75 & 0.02 & $<0.001$ \\
\hline ApoA2 (mmol/l) & 0.34 & 0.05 & 0.35 & 0.01 & 0.33 & 0.01 & 0.051 \\
\hline ApoB/ApoA1 & 0.66 & 0.29 & 0.88 & 0.04 & 0.46 & 0.01 & $<0.001$ \\
\hline ApoE (mg/l) & 56.43 & 29.08 & 71.69 & 4.93 & 42.13 & 2.03 & $<0.001$ \\
\hline $\operatorname{Lp}(a)(g / l)$ & 0.19 & 0.28 & 0.25 & 0.05 & 0.13 & 0.03 & 0.003 \\
\hline Frequencies & $n$ & $\%$ & $n$ & $\%$ & $\mathrm{n}$ & $\%$ & $p$ \\
\hline Men (\%) & 45 & 48.4 & 21 & 46.7 & 24 & 50 & 0.748 \\
\hline $\mathrm{AH}(\%)$ & 30 & 32.3 & 20 & 44.4 & 10 & 20.8 & 0.015 \\
\hline Abdominal obesity (\%) & 44 & 47.3 & 22 & 48.9 & 22 & 45.8 & 0.768 \\
\hline Smoking (\%) & 30 & 32.3 & 12 & 26.7 & 18 & 37.5 & 0.264 \\
\hline CHD history (\%) & 35 & 37.6 & 29 & 64.4 & 6 & 12.5 & $<0.001$ \\
\hline
\end{tabular}

Abbreviations: SD standard deviation, $T C$ total cholesterol, $L D L-C$ low-density lipoprotein cholesterol, $H D L-C$ high-density lipoprotein cholesterol, $T G$ triglycerides, $A p o$ apolipoprotein, $L p(a)$ lipoprotein(a), $A H$ arterial hypertension, $C H D$ coronary heart disease 


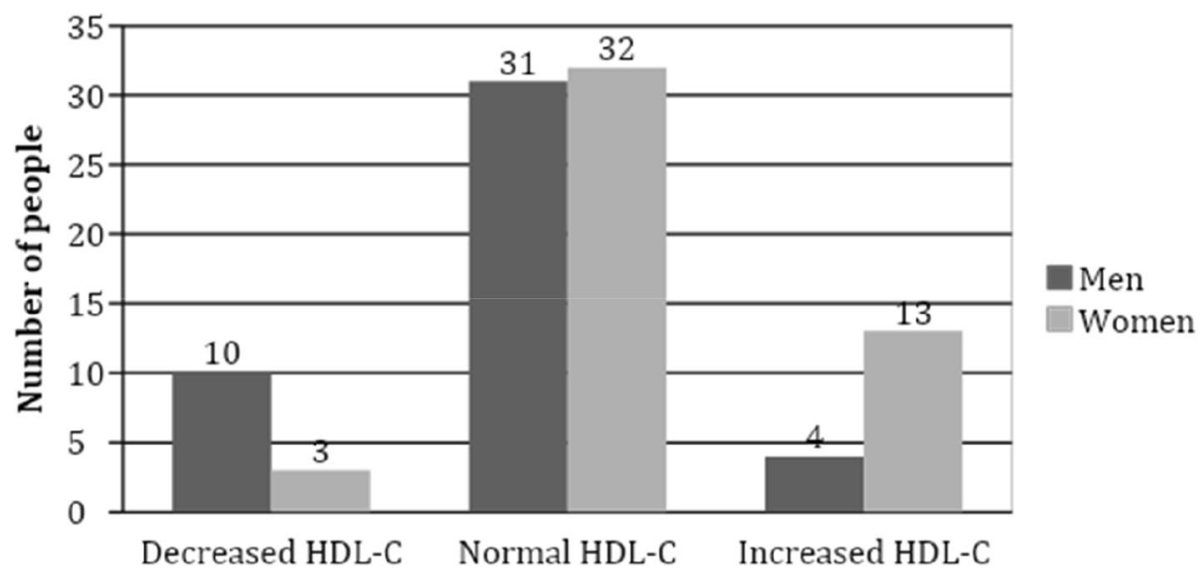

Fig. 1 Association between HDL-C concentration and gender in the study population, $p=0,015(n=93)$. Decreased HDL-C concentration was defined as $<1.08 \mathrm{mmol} / \mathrm{l}$, normal HDL-C concentration 1.08-1.4 and increased HDL-C concentration > 1.40. Decreased HDL-C was more frequently found among men

who did not consume alcoholic beverages $(p=0.004)$ (Fig. 3). No association was found between HDL-C concentration and cardiovascular risk factors, such as abdominal obesity, arterial hypertension, smoking and familial history of coronary heart disease. Although physically active subjects tended to have normal levels of HDL-C more frequently than participants with insufficient physical activity, no significant differences were found.

Strong statistically significant positive correlation between HDL-C and ApoA1 concentration was found in participants with SD $(r=0.63)$ and control group $(r=$ 0.866 ) (Table 2). The correlation was significant in all age groups. Higher HDL-C was also associated with higher LDL-C concentration in participants with SD $(r=0.412)$ and higher ApoA2 concentration in participants with SD $(r=0.418)$ and control group $(r=0.41)$.
HDL-C concentration was inversely associated with ApoE $(r=-0.314)$ and HDL-C efflux capacity $(r=-$ $0.335)$ in participants with SD. In addition, there was inverse association between HDL-C and TG concentration in men with SD $(r=-0.582)$ and control group $(r=-$ 0.38 ) in all age groups, and APOB/APOA1 ratio in control group $(r=-0.567)$; this association decreased with age (Table 3). Lower HDL-C was also associated with higher BMI in participants with SD $(r=-0.531)$ and control group $(r=-0.327)$ and higher waist circumference in women with SD $(r=-0.309)$. Age did not impact HDL-C concentration in this analysis.

\section{HDL cholesterol efflux capacity}

The average HDL-C efflux capacity in the study population was $47.5 \%$. Below-average HDL-C efflux capacity

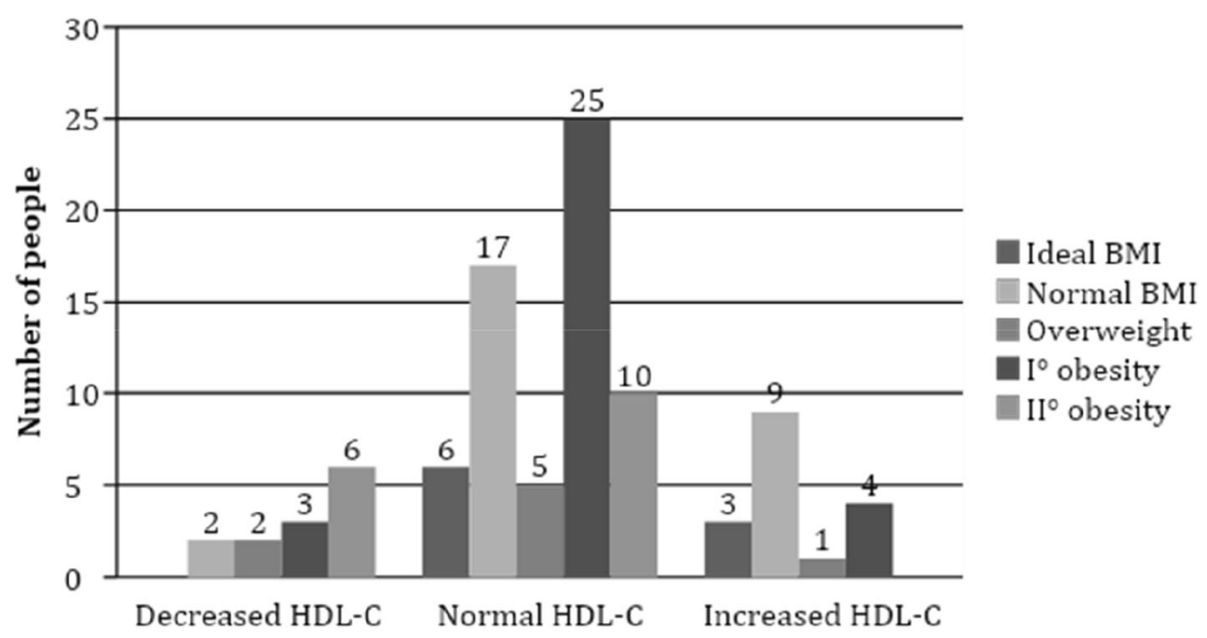

Fig. 2 Association between HDL-C concentration and BMl in the study population, $p=0.02(n=93)$. Ideal BMl -22 , normal $-20-25$ for men and 18.5-24 for women, overweight - 25-29.9, $1^{\circ}$ obesity - 30-40 and $I^{\circ}$ obesity - > 40. Normal HDL-C concentration was more often found in participants with $I^{\circ}$ obesity $(p=0.02)$ 


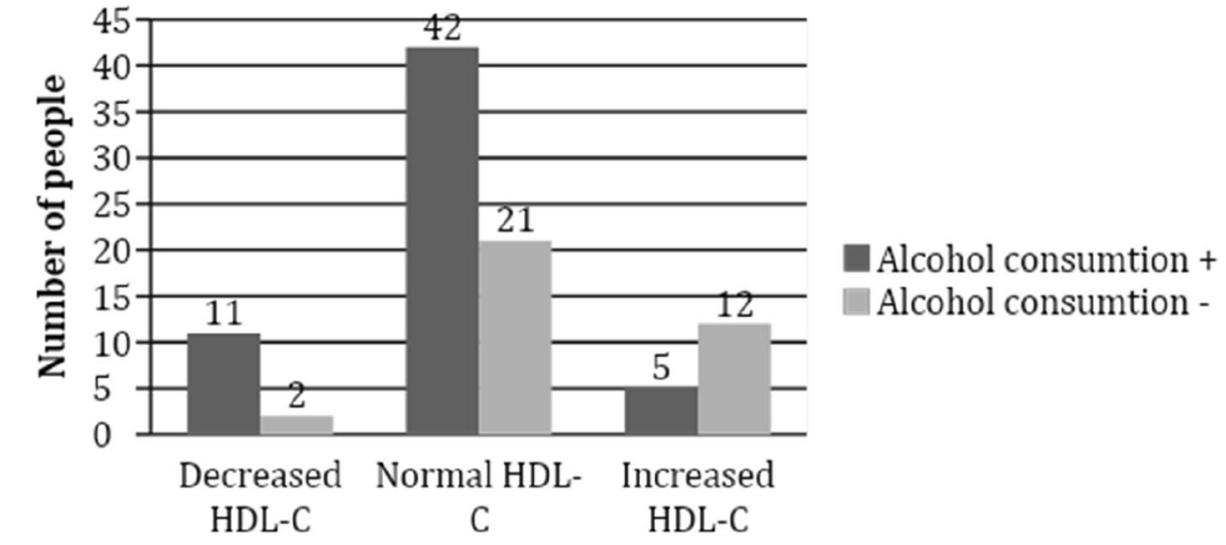

Fig. 3 Association between HDL-C concentration and alcohol consumption in the study population, $p=0.004$ ( $n=93)$. Normal HDL-C concentration was more often found in participants who consumed alcohol, in comparison with those who did not consume alcoholic beverages $(p=0.004)$

was found in $67.7 \%(N=63)$ of the participants (Fig. 4). Significant differences in HDL-C efflux capacity between men and women were not detected. Women with SD more often had below-average HDL-C efflux capacity ( $75 \%(N=36)$ vs $60 \%(N=27), p=0.238)$, however the difference was not statistically significant.

Statistically significant negative correlation between HDL-C efflux capacity and HDL-C concentration was found $(r=-0.228)$. Correlation was not significant when evaluated separately in men and women cohorts. No

Table 2 Spearman's correlation coefficients between HDL-C concentration and other characteristics in groups with and without severe dyslipidemia

\begin{tabular}{lllll}
\hline Characteristics & \multicolumn{3}{l}{$\mathrm{HDL}-\mathrm{C}(\mathrm{mmol} / \mathrm{l})$} \\
\cline { 2 - 5 } & $\mathrm{SD}-$ & $\mathrm{SD}+$ & $\mathrm{SD}+$ & \\
\cline { 4 - 5 } & & & $\mathrm{Men}$ & Women \\
\hline HDL-C efflux capacity (\%) & -0.146 & $-0.335^{*}$ & -0.123 & -0.198 \\
Age (years) & 0.130 & 0.061 & 0.059 & -0.030 \\
TC (mmol/l) & $0.342^{*}$ & 0.277 & -0.008 & $0.327^{*}$ \\
TG (mmol/l) & $-0.380^{*}$ & $-0.608^{*}$ & $-0.582^{*}$ & -0.217 \\
LDL-C (mmol/l) & -0.057 & $0.412^{*}$ & 0.083 & 0.232 \\
Apo A1 (mmol/l) & $0.866^{*}$ & $0.630^{*}$ & $0.713^{*}$ & $0.755^{*}$ \\
Apo B (mmol/l) & -0.097 & 0.275 & -0.065 & 0.126 \\
Apo A2 (mmol/l) & $0.410^{*}$ & $0.418^{*}$ & $0.317^{*}$ & $0.455^{*}$ \\
Apo E (mmol/l) & 0.140 & $-0.314^{*}$ & -0.160 & 0.050 \\
Apo B/Apo A1 & $-0.567^{*}$ & -0.015 & $-0.325^{*}$ & -0.104 \\
Lp(a) (g/l) & -0.263 & 0.232 & -0.141 & -0.015 \\
BMl (kg/m2) & $-0.327^{*}$ & $-0.531^{*}$ & $-0.461^{*}$ & $-0.441^{*}$ \\
WC (cm) & 0.183 & $-0.583^{*}$ & -0.189 & $-0.309^{*}$ \\
\hline
\end{tabular}

Abbreviations: HDL-C high-density lipoprotein cholesterol, TC total cholesterol, $T G$ triglycerides, LDL-C low-density lipoprotein cholesterol, Apo apolipoprotein, $L p(a)$ lipoprotein(a), $B M I$ body mass index, WC waist circumference

* Statistically significant as $p<0.05$ significant correlation between SD and HDL-C efflux capacity was found.

Below-average HDL-C efflux capacity was more frequently found in participants with normal BMI and class I obesity compared to overweight participants and those with class II obesity $(p \leq 0.05)$. It was observed that men with SD and normal weight had impaired HDL-C efflux capacity more frequently. No relation was found between HDL-C efflux capacity and participants' gender or cardiovascular risk factors, such as abdominal obesity, arterial hypertension, familial history of coronary heart disease and alcohol consumption. Although belowaverage HDL-C efflux capacity was more common

Table 3 Spearman's correlation coefficients between HDL-C concentration and other characteristics in different age groups

\begin{tabular}{llll}
\hline Characteristics & \multicolumn{3}{l}{ HDL-C (mmol/l) } \\
\cline { 2 - 4 } & $<45 y$ & $45-54 y$ & $\geq 55 y$ \\
\hline HDL-C efflux capacity (\%) & -0.217 & -0.267 & 0.006 \\
Age (years) & -0.330 & 0.282 & -0.365 \\
TC (mmol/l) & 0.110 & 0.226 & -0.177 \\
TG (mmol/l) & $-0.588^{*}$ & $-0.326^{*}$ & $-0.775^{*}$ \\
LDL-C (mmol/l) & 0.032 & 0.168 & -0.083 \\
Apo A1 (mmol/l) & $0.864^{*}$ & $0.669^{*}$ & $0.803^{*}$ \\
Apo B (mmol/l) & 0.127 & 0.065 & -0.349 \\
Apo A2 (mmol/l) & -0.014 & $0.600^{*}$ & 0.511 \\
Apo E (mg/l) & -0.108 & 0.040 & -0.481 \\
Apo B/Apo A1 & $-0.374^{*}$ & -0.143 & -0.535 \\
Lp(a) (g/l) & -0.295 & 0.138 & 0.194 \\
BMl (kg/m2) & $-0.561^{*}$ & $-0.400^{*}$ & -0.398 \\
WC (cm) & -0.272 & -0.273 & -0.209 \\
\hline
\end{tabular}

Abbreviations: HDL-C high-density lipoprotein cholesterol, TC total cholesterol, TG triglycerides, LDL-C low-density lipoprotein cholesterol, Apo apolipoprotein, $L p(a)$ lipoprotein(a), BMI body mass index, WC waist circumference

* Statistically significant as $p<0.05$; 


\section{HDL-C efflux capacity}

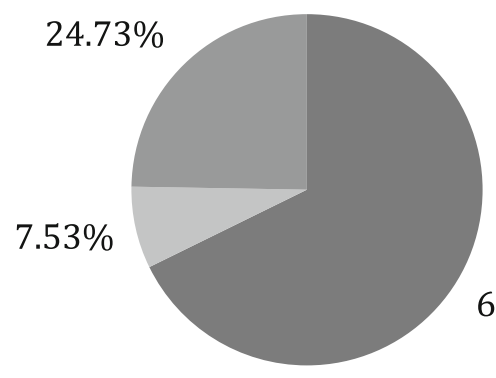

$67.74 \%$

Below average $\square$ Average $\square$ Above average

Fig. 4 Evaluation of HDL-C efflux capacity (\%) in the study population $(n=93)$. Majority of participants $(67.74 \%)$ had below average HDL-C efflux capacity

among physically active subjects compared to patients with insufficient physical activity, this difference was not statistically significant.

\section{Discussion}

According to LitHiR Primary Prevention Program, the prevalence of any type of dyslipidemia in middle-aged population (50-65 years old women and 40-55 years old men) is high $-89.7 \%$ [3], while the prevalence of low HDL-C is unexpectedly low - $13.7 \%$ [4]. It was estimated that average HDL-C concentration is $1.54 \pm 0.46 \mathrm{mmol} / \mathrm{l}$ $(59.6 \pm 17.8 \mathrm{mg} / \mathrm{dL})$ [3]. While in Poland NATPOL 2011 representative 40-59 years sample, prevalence of low-HDL was $35.2 \%$ in men and $22.2 \%$ in women, average HDL was $1.22 \mathrm{mmol} / \mathrm{l}(47.3 \mathrm{mg} / \mathrm{dL})$ in men and $1.41 \mathrm{mmol} / \mathrm{l}(54.7$ $\mathrm{mg} / \mathrm{dL}$ ) in women [20]. Interestingly participants of LitHiR with isolated low HDL-C had more favorable risk profile than participants with other lipid abnormalities, such as isolated triglyceridemia and atherogenic dyslipidemia [21]. In addition, recent studies suggest that low HDL-C may paradoxically be associated with reduced risk of incident CHD in black participants population [22], which led us to investigate the quality of HDL-C.

According to two prospective Danish cohort studies the HDL cholesterol concentration associated with the lowest risk of all-cause mortality is $1.9 \mathrm{mmol} / \mathrm{L}(73 \mathrm{mg} /$ $\mathrm{dL})$ for men, and $2.4 \mathrm{mmol} / \mathrm{L}(93 \mathrm{mg} / \mathrm{dL})$ for women [15]. Although some researchers define an HDL-C of $1.55 \mathrm{mmol} / \mathrm{l}(60 \mathrm{mg} / \mathrm{dL})$ or greater as high HDL-C and an HDL-C of $2.59 \mathrm{mmol} / \mathrm{l}(100 \mathrm{mg} / \mathrm{dL})$ or greater as very high HDL-C [23], there is no consensus on what HDL-C is "too high". In our study due to small sample size for statistical analysis sample was divided into 3 groups according to 33 percentiles and increased HDL-C was defined as HDL-C greater than $1.4 \mathrm{mmol} / \mathrm{l}(54 \mathrm{mg} / \mathrm{dL})$.
According to literature, HDL-C concentration is inversely associated with weight, abdominal circumference, TG concentration, number of small dense LDL particles, systemic inflammatory response and smoking [24]. Both extremely low and high HDL-C levels are associated with greater risk of all-cause, CHD and stroke mortalities and higher level of inflammatory factors [25], while the impact on risk of cancer is ambiguous [25], [22]. In our study low HDL-C was associated with higher BMI, abdominal circumference and TG. On the other hand, physical fitness is associated with higher HDL-C [26, 27]. This emphasizes the importance of lifestyle intervention in treating low HDL-C. It has been reported that 5-10\% weight loss can increase HDL-C approximately 8-10\% [28]. In our study physically active subjects tended to have normal levels of HDL-C more frequently than participants with insufficient physical activity, however this difference was not statistically significant.

Walter reported that plasma HDL-C levels change with age. In males HDL-C levels decrease during adolescence and early adulthood, but in elder age they are stable or even slightly increased [29]. In contrast, women's HDL-C levels remain stable throughout their lifetime, however, menopause often causes a slight decrease in HDL-C concentration [30]. In our study a weak but significant correlation between age and HDL-C concentration was found. However, the differences of HDL-C levels in different age groups of both men and women were statistically insignificant when calculated with ANOVA.

ApoA1 accounts for approximately $70 \%$ of HDL total protein mass, and ApoA2 for 15-20\%. Other proteins, such as ApoC, ApoD, ApoM and ApoA-IV, cholesteryl ester transfer protein (CEPT), and lipolytic enzymes, such as paraoxonase-1, glutathione peroxidase and plateletactivating factor acetylhydrolase (PAF-AH), as well as cholesterol acyl transferase form the remaining 15\% [31, 32]. ApoA1 plays a major role in reverse cholesterol transport and is a major anti-atherogenic and antioxidant factor of HDL-C [31]. ApoA2 is also associated with reverse cholesterol transport and antioxidant potential [33]. ApoE is responsible for receptor-mediated uptake of lipoprotein, antioxidant, anti-inflammatory and endothelial activity. It induces release of endothelial nitric oxide [31]. In our study HDL-C concentration was positively associated with ApoA1, ApoA2 and inversely associated with ApoE concentration and APOB/APOA1 ratio.

In this study we evaluated HDL-C efflux capacity in healthy participants and patients with severe dyslipidemia. It was found that the majority of participants (67.7\%) had below-average HDL-C efflux capacity. According to literature, there is a positive correlation between HDL-C efflux capacity and alcohol intake, and inverse correlation with type 2 diabetes and measures of obesity [18]. In our study HDL-C efflux capacity was not 
associated with traditional cardiovascular risk factors, except obesity (below-average HDL-C efflux capacity was more frequently found in participants with normal BMI and $\mathrm{I}^{\circ}$ obesity compared to overweight participants and those with $\mathrm{II}^{\circ}$ obesity). These are similar findings to Rohatgi et al. study, in which HDL cholesterol efflux capacity was minimally associated with cardiovascular risk factors [34]. However, our study did not reveal such tendencies. HDL-C efflux capacity was inversely associated with HDL-C concentration $(r=-0.228)$. Similar results were found in case control study of individuals with extremely high HDL-C and CAD [35].

It is still widespread myth in Lithuania that high HDL-C compensates effects of other lipid abnormalities. However even though average HDL-C concentration in Lithuanian middle age population is high, majority of participants in our study had below average HDL-C efflux capacity, which points out that HDL-C may be dysfunctional, which may contribute to residual cardiovascular disease risk. Even though HDL-C function is not yet a target in treatment of dyslipidemia, which limits application of this assay in everyday practice, we believe, that these novel findings of HDL-C function in Lithuanian population may contribute for future studies of new agents in dyslipidemia treatment.

It should be taken into account, that our results must be interpreted with some caution because this study has some potential limitations. First of all modest sample size led us to restriction of dividing population into subgroups and analyzing smaller variables and tendencies in between. Study population were found to be quite heterogeneous especially according age differences. In addition, the fact that some of participants were using statins may impact the results of our study. Further studies are needed.

\section{Conclusion}

Majority of participants had below-average HDL-C efflux capacity. It was inversely associated with HDL-C concentration. Abnormal HDL function may be associated with residual cardiovascular risk in Lithuanian population.

\section{Acknowledgements}

The authors would also like to thank Roma Puronaite for helping with statistical data analysis.

\section{Authors' contributions \\ SK, ZP, DK, AL, GM, JS, ASM, UG and ES designed the study, participated in the collecting and analyzing of the data, writing and reviewing the manuscript. MK reviewed the manuscript. All authors approved the final version of the manuscript for publication.}

\section{Funding}

This study did not receive funding.

\section{Availability of data and materials}

Data supporting the findings of this study are not publicly available, as the research is ongoing and further publications are being done.
Ethics approval and consent to participate

Study was approved of the Local Research Ethics Committee.

\section{Consent for publication}

Written informed consent for publication was obtained from each author, and there are no other persons who satisfy the criteria for authorship but are not listed.

\section{Competing interests}

The authors declare that they have no competing interests.

\section{Author details}

${ }^{1}$ Faculty of Medicine, Vilnius University, Santariškių 2, LT-08661 Vilnius, Lithuania. ${ }^{2}$ Faculty of Medicine, Clinic of Cardiac and Vascular Diseases, Vilnius University, Vilnius, Lithuania. ${ }^{3}$ Vilnius University Hospital Santaros Klinikos, Vilnius, Lithuania. ${ }^{4}$ Faculty of Medicine, Department of Physiology, Biochemistry, Microbiology and Laboratory Medicine, Vilnius University, Vilnius, Lithuania.

Received: 12 March 2019 Accepted: 30 September 2019

Published online: 26 October 2019

\section{References}

1. Country Health Profiles - Public Health - European Commission [Internet]. Public Health. [cited 2018 Oct 27]. Available from: https://ec.europa.eu/ health/sites/health/files/state/docs/chp_It_english.pdf.

2. EUROSTAT Cardiovascular diseases statistics [Internet]. Available from: https://ec.europa.eu/eurostat/statistics-explained/index.php/Cardiovascular_ diseases_statistics\#Deaths_from_cardiovascular_diseases.

3. The prevalence of dislipidemia and its relation to other risk factors: a nationwide survey of Lithuania | Request PDF [Internet]. ResearchGate. [cited 2018 Oct 27]. Available from: https://www.researchgate.net/ publication/282001927_The_prevalence_of_dislipidemia_and_its_relation to_other_risk_factors_a_nationwide_survey_of_Lithuania.

4. Kutkiene $S$, Petrulioniene $Z$, Laucevicius A, Serpytis $P$, Kasiulevicius $V$, Staigyte J, et al. Cardiovascular risk assessment of dyslipidemic middle-aged adults without overt cardiovascular disease over the period of 2009-2016 in Lithuania. Lipids Health Dis [Internet]. 2018 Oct 11 [cited 2018 Nov 28];17. Available from: https://www.ncbi.nlm.nih.gov/pmc/articles/PMC6180581/.

5. Lilley JS, Linton MF, Fazio S. Genetic disorders of HDL metabolism. Contemp Endocrinol. 2015:1:221-33.

6. Choi HY, Hafiane A, Schwertani A, Genest J. High-density lipoproteins: biology, epidemiology, and clinical management. Can J Cardiol. 2017;33(3):325-33.

7. Prospective Studies Collaboration, Lewington S, Whitlock G, Clarke R, Sherliker P, Emberson J, et al. Blood cholesterol and vascular mortality by age, sex, and blood pressure: a meta-analysis of individual data from 61 prospective studies with 55,000 vascular deaths. Lancet Lond Engl. 2007; 370(9602):1829-39.

8. Emerging Risk Factors Collaboration, Di Angelantonio E, Sarwar N, Perry P, Kaptoge S, Ray KK, et al. Major lipids, apolipoproteins, and risk of vascular disease. JAMA. 2009;302(18):1993-2000.

9. Linsel-Nitschke $P$, Tall AR. HDL as a target in the treatment of atherosclerotic cardiovascular disease. Nat Rev Drug Discov. 2005:4(3):193-205.

10. Colantonio LD, Bittner V, Reynolds K, Levitan EB, Rosenson RS, Banach M, et al. Association of Serum Lipids and Coronary Heart Disease in contemporary observational studies. Circulation. 2016;133(3):256-64.

11. Voight BF, Peloso GM, Orho-Melander M, Frikke-Schmidt R, Barbalic M, Jensen MK, et al. Plasma HDL cholesterol and risk of myocardial infarction: a mendelian randomisation study. Lancet. 2012:380(9841):572-80.

12. Keene D, Price C, Shun-Shin MJ, Francis DP. Effect on cardiovascular risk of high density lipoprotein targeted drug treatments niacin, fibrates, and CETP inhibitors: meta-analysis of randomised controlled trials including 117411 patients. BMJ. 2014;349:94379.

13. Toth PP, Barylski M, Nikolic D, Rizzo M, Montalto G, Banach M. Should low high-density lipoprotein cholesterol (HDL-C) be treated? Best Pract Res Clin Endocrinol Metab. 2014;28(3):353-68.

14. Otocka-Kmiecik A, Mikhailidis DP, Nicholls SJ, Davidson M, Rysz J, Banach M. Dysfunctional HDL: a novel important diagnostic and therapeutic target in cardiovascular disease? Prog Lipid Res. 2012;51(4):314-24.

15. Madsen CM, Varbo A, Nordestgaard BG. Extreme high high-density lipoprotein cholesterol is paradoxically associated with high mortality in 
men and women: two prospective cohort studies. Eur Heart J. 2017;38(32): 2478-86.

16. Navab M, Reddy ST, Van Lenten BJ, Fogelman AM. HDL and cardiovascular disease: atherogenic and atheroprotective mechanisms. Nat Rev Cardiol. 2011;8(4):222-32.

17. Rader DJ, Alexander ET, Weibel GL, Billheimer J, Rothblat GH. The role of reverse cholesterol transport in animals and humans and relationship to atherosclerosis. J Lipid Res. 2009;50(Supplement):S189-94.

18. Saleheen D, Scott R, Javad S, Zhao W, Rodrigues A, Picataggi A, et al. Association of HDL cholesterol efflux capacity with incident coronary heart disease events: a prospective case-control study. Lancet Diabetes Endocrinol. 2015 Jul;3(7):507-13.

19. National Cholesterol Education Program (NCEP) Expert Panel on Detection Evaluation, and Treatment of High Blood Cholesterol in Adults (Adult Treatment Panel III). Third Report of the National Cholesterol Education Program (NCEP) Expert Panel on Detection, Evaluation, and Treatment of High Blood Cholesterol in Adults (Adult Treatment Panel III) final report. Circulation. 2002;106(25):3143-421.

20. Zdrojewski T, Solnica B, Cybulska B, Bandosz P, Rutkowski M, Stokwiszewski J, et al. Prevalence of lipid abnormalities in Poland. The NATPOL 2011 survey. Kardiol Pol. 2016;74(3):213-23.

21. Kutkiene S, Petrulioniene Z, Laucevicius A, Matuzeviciene G, Kasiulevicius V Petrulionyte $E$, et al. Cardiovascular risk profile of patients with atherogenic dyslipidemia in middle age Lithuanian population. Lipids Health Dis. 2018; 17(1):208.

22. Penson P, Long DL, Howard G, Howard VJ, Jones SR, Martin SS, et al. Associations between cardiovascular disease, cancer, and very low highdensity lipoprotein cholesterol in the REasons for geographical and racial differences in stroke (REGARDS) study. Cardiovasc Res. 2019;115(1):204-12.

23. Toth PP, Barter PJ, Rosenson RS, Boden WE, Chapman MJ, Cuchel M, et al. High-density lipoproteins: a consensus statement from the National Lipid Association. J Clin Lipidol. 2013;7(5):484-525.

24. Cooney MT, Dudina A, De Bacquer D, Wilhelmsen L, Sans S, Menotti A, et al. HDL cholesterol protects against cardiovascular disease in both genders, at all ages and at all levels of risk. Atherosclerosis. 2009;206(2):611-6.

25. Mazidi M, Mikhailidis DP, Banach M. Associations between risk of overall mortality, cause-specific mortality and level of inflammatory factors with extremely low and high high-density lipoprotein cholesterol levels among American adults. Int J Cardiol. 2019;276:242-7.

26. Mertens E, Clarys P, Lefevre J, Charlier R, Knaeps S, Deforche B. Longitudinal study on the association between cardiorespiratory fitness, anthropometric parameters and blood lipids. J Phys Act Health. 2016;13(5):467-73.

27. Demmer DL, Beilin LJ, Hands B, Burrows S, Cox KL, Oddy WH, et al. Fatness and Fitness With Cardiometabolic Risk Factors in Adolescents. J Clin Endocrinol Metab. 2017;102(12):4467-76.

28. Vega GL, Grundy SM, Barlow CE, Leonard D, Willis BL, DeFina LF, et al. Association of triglyceride-to-high density lipoprotein cholesterol ratio to cardiorespiratory fitness in men. J Clin Lipidol. 2016;10(6):1414-22 e1.

29. Walter M. Interrelationships among HDL metabolism, aging, and atherosclerosis. Arterioscler Thromb Vasc Biol. 2009:29(9):1244-50.

30. Kreisberg RA, Kasim S. Cholesterol metabolism and aging. Am J Med. 1987;82(1):54-60

31. Arora S, Patra SK, Saini R. HDL-A molecule with a multi-faceted role in coronary artery disease. Clin Chim Acta Int J Clin Chem. 2016;452:66-81.

32. Podrez EA. Anti-oxidant properties of high-density lipoprotein and atherosclerosis. Clin Exp Pharmacol Physiol. 2010;37(7):719-25.

33. Rotllan N, Ribas V, Calpe-Berdiel L, Martín-Campos JM, Blanco-Vaca F, EscolàGil JC. Overexpression of human apolipoprotein A-II in transgenic mice does not impair macrophage-specific reverse cholesterol transport in vivo. Arterioscler Thromb Vasc Biol. 2005;25(9):e128-32.

34. Rohatgi A, Khera A, Berry JD, Givens EG, Ayers CR, Wedin KE, et al. HDL cholesterol efflux capacity and incident cardiovascular events. N Engl J Med. 2014;371(25):2383-93.

35. Agarwala AP, Rodrigues A, Risman M, McCoy M, Trindade K, Qu L, et al. High-density lipoprotein (HDL) phospholipid content and cholesterol efflux capacity are reduced in patients with very high $\mathrm{HDL}$ cholesterol and coronary disease. Arterioscler Thromb Vasc Biol. 2015;35(6):1515-9.

\section{Publisher's Note}

Springer Nature remains neutral with regard to jurisdictional claims in published maps and institutional affiliations.

Ready to submit your research? Choose BMC and benefit from:

- fast, convenient online submission

- thorough peer review by experienced researchers in your field

- rapid publication on acceptance

- support for research data, including large and complex data types

- gold Open Access which fosters wider collaboration and increased citations

- maximum visibility for your research: over $100 \mathrm{M}$ website views per year

At BMC, research is always in progress.

Learn more biomedcentral.com/submissions 